\title{
Whole-Genome Sequencing of Drug-Resistant Mycobacterium tuberculosis Strains, Tunisia, 2012-2016
}

\author{
Imen Bouzouita, Andrea Maurizio Cabibbe, \\ Alberto Trovato, Henda Daroui, Asma Ghariani, \\ Basma Midouni, Leila Essalah, Emna Mehiri, \\ Daniela Maria Cirillo, Leila Slim Saidi
}

To investigate transmission of drug-resistant strains of $M y$ cobacterium tuberculosis in Tunisia, we performed wholegenome sequencing on 46 multidrug-resistant strains isolated during 2012-2016. Core-genome multilocus sequence typing grouped $30(65.2 \%)$ strains into 3 clusters, indicating extensive recent transmission and Haarlem clone predominance. Whole-genome sequencing might help public health services undertake appropriate control actions.

$\mathrm{T}$ he emergence of drug-resistant strains of Mycobacterium tuberculosis is hampering the control of tuberculosis (TB) worldwide. In Tunisia in 2017, the estimated percentage of TB patients with multidrug-resistant (MDR)/rifampinresistant TB was $1.1 \%$ among those with new infection and $13 \%$ among those with previously treated infection (1).

Effective and rapid tools are needed to characterize and track the transmission chains of MDR/rifampin-resistant TB. Whole-genome sequencing (WGS) has shown higher discriminatory power for epidemiologic investigations than have other conventional genotyping methods (e.g., spoligotyping, IS6110 restriction fragment length polymorphism, and mycobacterial interspersed repetitive unit-variable-number tandem repeat). Indeed, WGS has enabled investigators to rule out false transmission events (2-7). Furthermore, WGS enables simultaneous determination of polymorphisms and insertions/deletions linked to resistance to first-line and second-line drugs (8).

In this study, we used WGS to investigate transmission of MDR and extensively drug resistant (XDR) TB strains isolated in Tunisia over a 4-year period by applying the core-genome multilocus sequence typing (cgMLST)

Author affiliations: National Reference Laboratory for Mycobacteria, Ariana, Tunisia (I. Bouzouita, H. Daroui, A. Ghariani, B. Midouni, L. Essalah, E. Mehiri, L. Slim Saidi); University of Tunis El Manar, Tunis, Tunisia (I. Bouzouita, B. Midouni); San Raffaele Scientific Institute, Milan, Italy (A.M. Cabibbe, A. Trovato, D.M. Cirillo); University of Monastir, Monastir, Tunisia (A. Ghariani, E. Mehiri, L. Slim Saidi)

DOI: https://doi.org/10.3201/eid2503.181370 scheme and identifying the drug-resistance marker for first-line and second-line drug resistance. This study was approved by the ethics committee of A. Mami Pneumology Hospital, Ariana, Tunisia.

\section{The Study}

We retrospectively studied $46 \mathrm{MDR} M$. tuberculosis isolates collected from $46 \mathrm{HIV}$-negative patients in Tunisia during June 2012-June 2016, which represented 57 (80.7\%) cases of MDR TB. Of the 46 isolates, 6 represented all (100\%) XDR TB cases recorded in the country during that period. We performed drug-susceptibility testing for resistance to first-line drugs (except pyrazinamide) by using the proportion method on Lowenstein-Jensen medium. For pyrazinamide and second-line drugs, we performed drug-susceptibility testing on a Bactec MGIT 960 system (Becton, Dickinson and Company, http://www.bd.com). WGS was performed on the MiniSeq platform (Illumina Inc., https:// www.illumina.com) targeting a minimum average reads coverage of 50-fold. To analyze the mutations involved in drug resistance and related to lineage determination, we used PhyResSe and TGS TB $(9,10)$. We performed the cgMLST scheme version 2.1, considering 2,891 core genes, by using Ridom SeqSphere+ version 5.0.0 software (Ridom $\mathrm{GmbH}$,_https://www.cgmlst.org) (7). To define a strain as a part of a recent transmission chain, we fixed a threshold of $<6$ allele variants. For statistical analyses, we calculated $p$ values by using OpenEpi version 3 (https://www.openepi. com) and considered $\mathrm{p}<0.05$ to be significant.

Patient origins are reported in the Appendix (https:// wwwnc.cdc.gov/EID/article/25/3/18-1370-App1.xlsx). Most MDR TB cases $(19 ; 41.3 \%)$ were recorded in the Bizerte region in northern Tunisia. WGS revealed that all MDR/XDR strains belonged to the European-American lineage (lineage 4); the Haarlem family was the most frequent $(71.8 \%)$ (Figure 1). (WGS files from this study have been submitted to the European Nucleotide Archive as fastq files under study accession no. PRJEB30463, https:// www.ebi.ac.uk/ena/data/search?query=PRJEB30463).

The cluster analysis (Figure 2) showed that $30(65.2 \%)$ of 46 isolates were grouped within 3 clusters, and most (90.0\%) clustered MDR TB cases belonged to the Haarlem family (Figure 1). Of note, a big cluster of 24 MDR strains linked to Haarlem (cluster 1) was detected over 
Figure 1. Lineage and family distribution of drug-resistant strains identified during study of drug-resistant Mycobacterium tuberculosis, Tunisia, 2012-2016. A) MDR strains. Haarlem was the most frequently represented family $(71.8 \%)$ among MDR strains. Among other samples, $21.7 \%$ belonged to the LAM family, $4.3 \%$ presented other genotypes of the EA lineage, and $2.2 \%$ the Uganda genotype. B) Clustered MDR strains. Haarlem
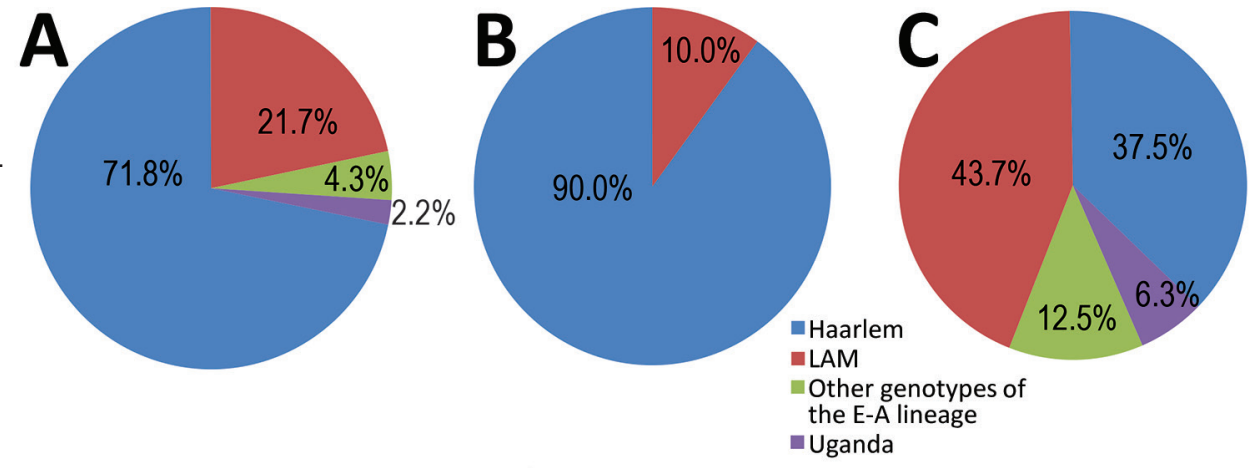
was also the most frequent family (90.0\%); the rest belonged to the LAM family. C) Nonclustered MDR strains. Among nonclustered isolates, LAM was the most frequently represented family $(43.7 \%)$, followed by Haarlem, which was detected in $37.5 \%$ of the strains. EA, European-American lineage; LAM, Latin-American-Mediterranean lineage; MDR, multidrug-resistant.

the entire study period. We found no significant associations between this cluster and patient sex, age $(\leq 35$ years, $>35$ years), and resistance to second-line drugs (XDR TB) $(\mathrm{p}>0.05)$; however, we found a significant association with this cluster and pyrazinamide resistance $(\mathrm{p}=0.001)$ and with Bizerte $(p=0.014)$. Despite the association with the Bizerte region, patients from various regions were part of this cluster (Figure 2). We also found a significant association between cluster 2 (Haarlem) and Beja $(p<0.001)$ and between cluster 3 (Latin-American-Mediterranean) and Ben Arous $(p<0.001)$. Epidemiologic links were confirmed for patients in these 2 transmission chains (Table 1).

All cluster 1 strains shared mutations in drug-resistance genes (e.g., rpoB, Ser450Leu, the compensatory

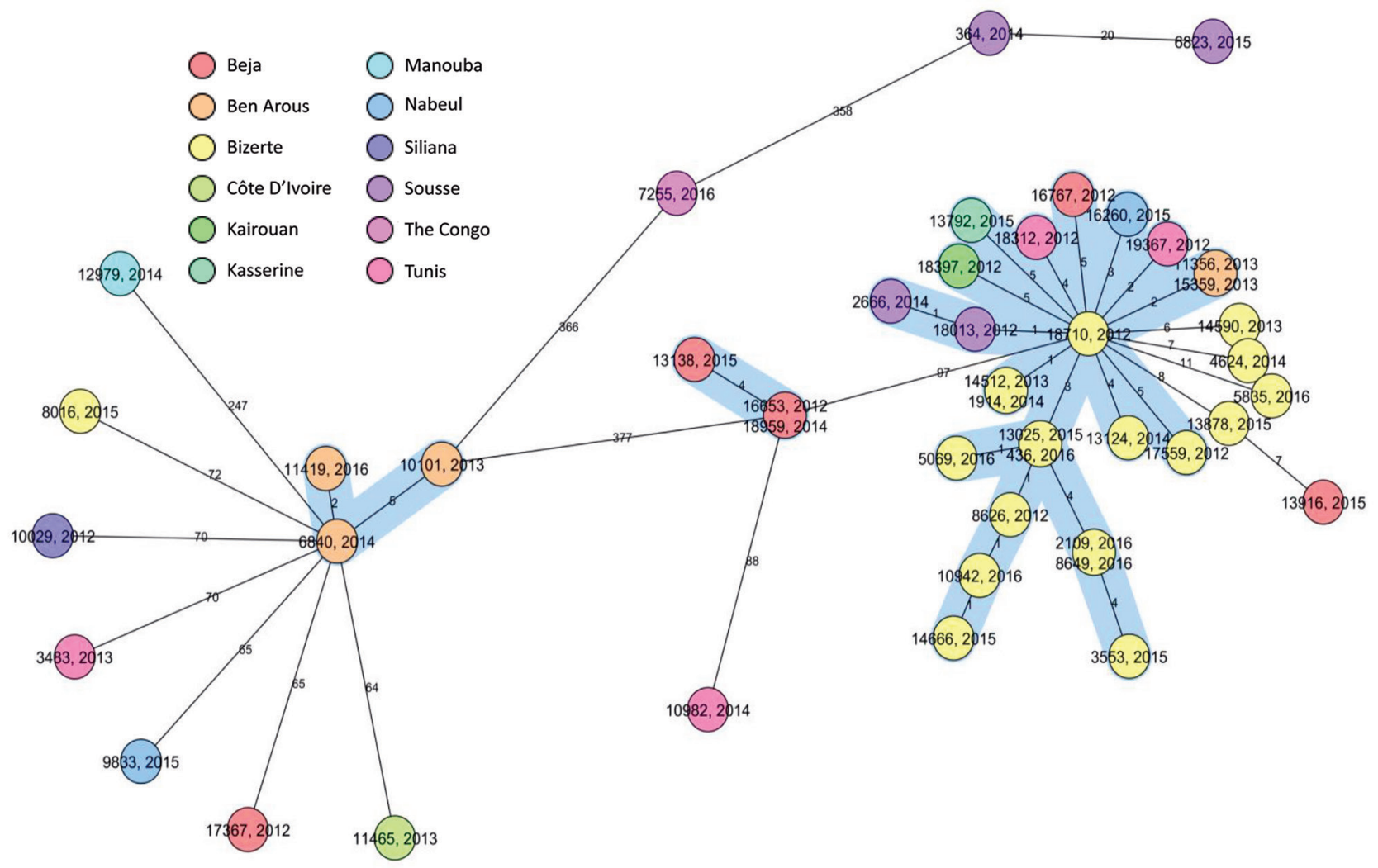

Figure 2. Core-genome multilocus sequence typing-based minimum spanning tree for strains identified during study of drug-resistant Mycobacterium tuberculosis, Tunisia, 2012-2016. Ridom SeqSphere+ minimum spanning tree for 46 samples based on 2,891 columns, pairwise ignoring missing values, logarithmic scale. Cluster distance threshold $=5$ alleles. Colors indicate regions and countries of patient origin. 
Table 1. Epidemiologic information about clustered cases identified during study of drug-resistant Mycobacterium tuberculosis strains, Tunisia, 2012-2016*

\begin{tabular}{|c|c|c|c|}
\hline Cluster, group & pncA mutation & $\begin{array}{c}\text { No. } \\
\text { patients }\end{array}$ & Epidemiologic links \\
\hline \multicolumn{4}{|l|}{ Cluster 1, Haarlem† } \\
\hline Group 1 & Gln10His & 9 & $\begin{array}{c}\text { Confirmed for patients } 10942,14666 \text { ( } 2 \text { brothers with XDR TB), } \\
8626 \text { (their neighbor with XDR TB) in Bizerte, and patients } \\
13025 \text { and } 2109 \text { (2 friends from Bizerte); probable for patients } \\
3553,436,5069,8649 \text { (from Bizerte) }\end{array}$ \\
\hline Group 2 & Leu4Trp & 5 & $\begin{array}{c}\text { Probable for patients } 18013 \text { and } 2666 \text { (from Sousse, } \\
\text { the central part of Tunisia) }\end{array}$ \\
\hline Group 3 & $A-11 C$ & 4 & Not available \\
\hline Group 4 & CA deletion $(+339,+340)$ & 2 & $\begin{array}{l}\text { Probable for patients } 11356 \text { and } 15359 \text { (from Ben Arous, } 10 \mathrm{~km} \text { from the } \\
\text { capital Tunis) }\end{array}$ \\
\hline Group 5 & Cys14Stop & 2 & Confirmed for patients 1914 and 14512 (brother and sister from Bizerte) \\
\hline Group 6 & Wild type & 2 & Not available \\
\hline Cluster 2, Haarlem & Wild type & 3 & $\begin{array}{l}\text { Confirmed for patients 13138, 18959, } 16653 \text { (same family: father, son, } \\
\text { and uncle; Beja; Northwest of Tunisia) }\end{array}$ \\
\hline Cluster 3, LAM & Wild type & 3 & Confirmed for patients $10101,11419,6840$ (neighbors from Ben Arous) \\
\hline
\end{tabular}

mutation rpoBVal534Met (11), katG Ser315Thr, embB Met306Ile, and gidBArg47Trp). However, diversity was noticed in $p n c A$ mutations conferring resistance to pyrazinamide. On the basis of $p n c A$ variation, this cluster was split into 6 groups (Table 1).

Four XDR isolates belonged to cluster 1. Three XDR isolates from 2 brothers (patients 10942 and 14666) and their neighbor (patient 8626) shared mutations conferring resistance to second-line drugs and to pyrazinamide (Tables 1,2), supporting evidence of direct transmission of this XDR strain. The fourth XDR TB case (patient/strain 13792) differed in mutations conferring resistance to pyrazinamide and fluoroquinolones. This case probably shared an ancestral MDR strain with cluster 1, which had evolved differently because of poor adherence of the patient to drug therapy (Table 2; Appendix). Five nonclustered MDR isolates linked to Haarlem (4 patients from Bizerte) were distant by $6-11$ alleles from cluster 1 showing the same polymorphisms in rpoB, $k a t G, e m b B$, and gidB (Figure 2; Appendix). Of note, cluster 2 presented the compensatory mutation $r p o B$ Val534Met, but no variation in $e m b B$, gidB, and $p n c A$ was detected (Appendix). This Haarlem cluster showed limited transmission compared with cluster 1 , which was distant by 97 alleles (Figure 2).

In our study, mutation rpoBSer450Leu was mostly associated with rifampin resistance $(\mathrm{n}=42,91.3 \%)$, whereas codon 315 of kat $G$ was most involved with isoniazid resistance $(\mathrm{n}=43,93.4 \%)$. Three nonclustered MDR isolates presented a genomic deletion of $\approx 10.4 \mathrm{~kb}$, which included the entire $k a t G$ gene (strain 3483) and 2 uncommon mutations in katG: Gly269Asp and Gly279Asp (patients/strains 9833, 10982) (Appendix). All pyrazinamide-resistant strains detected with the MGIT 960 system ( $\mathrm{n}=33,71.7 \%$ ) had a mutation in the $p n c A$ gene or its promoter.

Regarding second-line drugs, all 13 fluoroquinoloneresistant strains had a mutation in the quinolone resistancedetermining region of $g y r A$, gyrB, or both. For secondline injectable drugs, 4 XDR strains had the mutation $r r s$ A1401G and 2 had the mutation eis C-14T and a frame shift in tlyA (Table 2).

\begin{tabular}{|c|c|c|c|c|c|c|c|}
\hline $\begin{array}{l}\text { No. } \\
\text { strains }\end{array}$ & Cluster & $\begin{array}{c}\text { Resistance to FQ, AG, or } \\
\text { CAP on MGIT } 960\end{array}$ & gyrA & gyrB & rrs & tlyA & eis-p \\
\hline 3 & 1 & OFX, LVX, KAN, AMK, CAP & Asp94Gly† & & A1401G† & & \\
\hline 1 & 1 & OFX, LVX, KAN, AMK, CAP & & Asp461His/Gly470Cys & $\mathrm{A} 1401 \mathrm{G} \dagger$ & & \\
\hline 1 & 1 & OFX, LVX & Ala90Val† & & & & \\
\hline 1 & 1 & OFX, LVX & Asp94Gly† & & & & \\
\hline 1 & 3 & OFX, LVX & Ser91Pro† & & & & \\
\hline 1 & Not clustered & OFX, LVX, KAN & Asp94Ala† & Asp461Asn & & & $\mathrm{C}-14 \mathrm{~T} \dagger$ \\
\hline 1 & Not clustered & OFX, LVX, CAP & Ser91Pro† & & & INS of $2 \mathrm{G} \dagger$ & \\
\hline 1 & Not clustered & OFX, LVX & Asp94Ser & & & & \\
\hline 1 & Not clustered & OFX, LVX & Ala90Val† & & & & \\
\hline 1 & Not clustered & OFX, LVX & & Asp461Asn & & & \\
\hline 1 & Not clustered & OFX, LVX & Asp94Tyr† & & & & \\
\hline
\end{tabular}




\section{Conclusions}

cgMLST analysis showed that $65.2 \%$ of MDR/XDR strains of $M$. tuberculosis were clustered, reflecting extensive transmission in Tunisia, particularly of a Haarlem clone. This Haarlem clone showed polymorphisms rроB Ser450Leu, Val534Met, katG Ser315Thr, embB Met306Ile, and gidBArg47Trp, and in $p n c A$ genes previously identified in a Haarlem MDR TB outbreak in the Bizerte region during 2001-2011 $(13,14)$. As indicated by statistical association, we conclude that this cluster is still spreading in the Bizerte area. However, diffusion in different regions of the country is alarming and requires intensified efforts to control and diagnose drug-resistant TB.

Only 2 strains, belonging to cluster 1 , did not have any mutations in $p n c A$. The wild-type $p n c A$ isolates might represent the genotype of the first strains that emerged in Bizerte and evolved since 2001 by acquiring single-nucleotide polymorphisms in $p n c A$ and the other genes, including genes involved in resistance to second-line drugs. It has been reported that the mutation rate during a transmission chain or TB latency is not completely stable and is estimated at $0.3-0.5$ single-nucleotide polymorphisms/ genome/year $(4,5,15)$, which leads to increased numbers of allele variants for some isolates and might explain the results found for 5 MDR strains distant from cluster 1 by 6-11 alleles.

The main limitation of this study is the incomplete number of MDR TB cases ( $\approx 19 \%$ missing). Epidemiologic information to confirm all clustered cases is lacking.

In summary, cgMLST-based WGS showed extensive transmission of MDR/XDR TB in Tunisia over 4 recent years, thereby indicating that MDR TB is not fully controlled. Use of this molecular approach for surveillance purposes might enable the public health service to undertake appropriate control actions, particularly in specific settings of this country.

\section{Acknowledgments}

We thank Ibtissem Blanco, Rachid Fourati, and Dhikrayet Gamara for their contribution to this study.

\section{About the Author}

Ms. Bouzouita is a PhD student at the National Reference Laboratory for Mycobacteria, Ariana, Tunisia. Her research focuses on TB diagnosis, TB drug resistance, and molecular epidemiology of TB.

\section{References}

1. World Health Organization. Global Tuberculosis Report. Geneva: The Organization; 2018. p. 256.

2. Roetzer A, Diel R, Kohl TA, Rückert C, Nübel U, Blom J, et al. Whole genome sequencing versus traditional genotyping for investigation of a Mycobacterium tuberculosis outbreak: a longitudinal molecular epidemiological study. PLoS Med. 2013;10:e1001387. http://dx.doi.org/10.1371/journal.pmed.1001387

3. Nikolayevskyy V, Kranzer K, Niemann S, Drobniewski F. Whole genome sequencing of Mycobacterium tuberculosis for detection of recent transmission and tracing outbreaks: a systematic review. Tuberculosis (Edinb). 2016;98:77-85. http://dx.doi.org/10.1016/ j.tube.2016.02.009

4. Bryant JM, Schürch AC, van Deutekom H, Harris SR, de Beer JL, de Jager $\mathrm{V}$, et al. Inferring patient to patient transmission of Mycobacterium tuberculosis from whole genome sequencing data. BMC Infect Dis. 2013;13:110. http://dx.doi.org/10.1186/1471-2334-13-110

5. Walker TM, Ip CL, Harrell RH, Evans JT, Kapatai G, Dedicoat MJ, et al. Whole-genome sequencing to delineate Mycobacterium tuberculosis outbreaks: a retrospective observational study. Lancet Infect Dis. 2013;13:137-46. http://dx.doi.org/10.1016/ S1473-3099(12)70277-3

6. Hatherell HA, Colijn C, Stagg HR, Jackson C, Winter JR, Abubakar I. Interpreting whole genome sequencing for investigating tuberculosis transmission: a systematic review. BMC Med. 2016;14:21. http://dx.doi.org/10.1186/s12916-016-0566-x

7. Kohl TA, Diel R, Harmsen D, Rothgänger J, Walter KM, Merker M, et al. Whole-genome-based Mycobacterium tuberculosis surveillance: a standardized, portable, and expandable approach. J Clin Microbiol. 2014;52:2479-86. http://dx.doi.org/10.1128/JCM.00567-14

8. Papaventsis D, Casali N, Kontsevaya I, Drobniewski F, Cirillo DM, Nikolayevskyy V. Whole genome sequencing of Mycobacterium tuberculosis for detection of drug resistance: a systematic review. Clin Microbiol Infect. 2017;23:61-8. http://dx.doi.org/10.1016/ j.cmi.2016.09.008

9. Feuerriegel S, Schleusener V, Beckert P, Kohl TA, Miotto P, Cirillo DM, et al. PhyResSE: a Web tool delineating Mycobacterium tuberculosis antibiotic resistance and lineage from whole-genome sequencing data. J Clin Microbiol. 2015;53: 1908-14. http://dx.doi.org/10.1128/JCM.00025-15

10. Sekizuka T, Yamashita A, Murase Y, Iwamoto T, Mitarai S, Kato S, et al. TGS-TB: total genotyping solution for Mycobacterium tuberculosis using short read whole genome sequencing. PLoS One. 2015;10:e0142951. http://dx.doi.org/10.1371/journal.pone.0142951

11. Meftahi N, Namouchi A, Mhenni B, Brandis G, Hughes D, Mardassi $\mathrm{H}$. Evidence for the critical role of a secondary site $r p o B$ mutation in the compensatory evolution and successful transmission of an MDR tuberculosis outbreak strain. J Antimicrob Chemother. 2016;71:324-32. http://dx.doi.org/10.1093/jac/dkv345

12. Miotto P, Tessema B, Tagliani E, Chindelevitch L, Starks AM, Emerson C, et al. A standardised method for interpreting the association between mutations and phenotypic drug resistance in Mycobacterium tuberculosis. Eur Respir J. 2017;50:1701354. http://dx.doi.org/10.1183/13993003.01354-2017

13. Mardassi H, Namouchi A, Haltiti R, Zarrouk M, Mhenni B, Karboul A, et al. Tuberculosis due to resistant Haarlem strain, Tunisia. Emerg Infect Dis. 2005;11:957-61. http://dx.doi.org/ 10.3201/eid1106.041365

14. Dekhil N, Meftahi N, Mhenni B, Ben Fraj S, Haltiti R, Belhaj S, et al. MDR-TB outbreak among HIV-negative Tunisian patients followed during 11 years. PLoS One. 2016;11:e0153983. http://dx.doi.org/10.1371/journal.pone.0153983

15. Ford CB, Shah RR, Maeda MK, Gagneux S, Murray MB, Cohen T, et al. Mycobacterium tuberculosis mutation rate estimates from different lineages predict substantial differences in the emergence of drug-resistant tuberculosis. Nat Genet. 2013; 45:784-90. http://dx.doi.org/10.1038/ng.2656

Address for correspondence: Imen Bouzouita, National Reference Laboratory for Mycobacteria, Microbiology Laboratory,

A. Mami Pneumology Hospital, 2080 Ariana, Tunisia;

email: imen.bouzouita@hotmail.com 